\title{
Electromagnetic grazing anomalies. Energy flux extrema
}

\author{
A.V. Kats \\ Usikov Institute for Radiophysics and Electronics of National Academy of Sciences of Ukraine \\ 12 Acad. Proskuri, Kharkiv 61085, Ukraine \\ E-mail: avkats@ire.kharkov.ua
}

Received January 17, 2019, published online March 26, 2019

\begin{abstract}
The diffraction of electromagnetic waves at the surface periodic structures accompanied by strong anomalous effects in different diffraction orders is considered in detail for high-contrast interfaces. We restrict discussion by the transverse magnetic polarization of the incident wave (the magnetic field is orthogonal to the plane of incidence) and the simplest geometry when the plane of incidence is orthogonal to the grating grooves. The most attention is devoted to the strong maxima and minima of the energy flux density accompanying specific grazing propagation of some diffraction order. Relation to other anomalies, both Rayleigh and the resonance ones is discussed as well.
\end{abstract}

Keywords: diffraction, wave, anomaly, resonance, grating, flux.

\section{Introduction}

It has been well known since early 1900s that the light diffraction by metal gratings is accompanied by a number of strong spectral and angular anomalies which manifest themselves by the fast dependence of the intensities on the wavelength and/or angle of incidence. The pioneer' work on the subject was performed by R. Wood in 1902 [1] with metal gratings. The first physical interpretation of some of the observed peculiarities was presented by Lord Rayleigh in [2]. He associated them with the branch points related to diffracted waves (i.e., with the transitions from the outgoing wave to the evanescent one and vice versa in different diffracted orders). Such explanation is incomplete due to the fact that some Wood anomalies are to be attributed to the resonance excitation of surface electromagnetic wave at the metallair interface. Such interpretation was first proposed by U. Fano [3]. The resonantly excited waves are called the surface plasmon polaritons (SPPs) [4]. The resonance anomaly is still widely discussed due to its perspective role in nanophotonics. Later, Wood caught site of one more anomaly relating to anomalously high intensity of the grazing outgoing wave: “...the spectrum leaving at grazing emergence, which is the one which governs the appearance of the anomalous bands, is very bright" [5]. Below the anomalies attributed to the grazing propagating waves are referred to as GA (Grazing Anomaly).
It is of essence that the Rayleigh anomaly exists for an arbitrary interface and polarization. However, it is much more pronounced for the high-dielectric contrast interface and for TM (transverse magnetic) polarization if the media we are dealing with are nonmagnetic. In what follows we restrict the consideration to the nonmagnetic case only. The results for the magnetic case can be obtained by replacing the dielectric permittivity, $\varepsilon$, with the magnetic permeability, $\mu$, and the TM polarization by the TE one and vice versa. The resonance anomaly can exist only for such interfaces that support surface electromagnetic waves (SEW). GA anomaly is rather universal and is well expressed for high contrast interfaces for TM polarization. To our knowledge it was first discussed theoretically in [6].

Consider the main properties of these anomalies. The branch (Rayleigh) point anomaly is of general type, its position can be easily obtained from the Bragg diffraction conditions and it exists for arbitrary polarization and interfaces. However, it is more expressed for metals under TM polarization. At the Rayleigh point the derivative of the diffracted wave intensity with respect to the wavelength and angle of incidence turns infinity. The resonance anomaly is less general because it is caused by existence of welldefined eigenmodes of the interface. ${ }^{*}$

For isotropic and nonmagnetic dissipation-free media such surface-localized electromagnetic waves do exist under the conditions $\varepsilon<0, \varepsilon_{d}>0, \varepsilon_{d}+\varepsilon<0$, where $\varepsilon$ and

* We restrict consideration to interface of two homogeneous isotropic nonmagnetic media, say, metal and dielectric. If between these two media exists some third one (even very thin layer), then additional to SPP resonances can occur [7]. For anysotropic media the resonance can be caused by other than SPP surface modes, say, Dyakonov ones, see [8] and citations therein. 
$\varepsilon_{d}$ denote dielectric permittivity of the metal and the adjacent dielectric, respectively. The SPP in-plane wavenumber $Q=Q(\omega)=\frac{\omega}{c} \sqrt{\varepsilon \varepsilon_{d} /\left(\varepsilon+\varepsilon_{d}\right)}>0$, where $\omega$ is the (angular) frequency of the incident wave, exceeds the wavenumber of the adjacent dielectric volume wave with the same frequency, $k=k(\omega)=\omega \sqrt{\varepsilon_{d}} / c, Q>k$. The square root symbol stays for the main branch, so that $\sqrt{Z}=\sqrt{|Z|} \exp (i \phi / 2)$ for $Z=|Z| \exp (i \phi)$ with $\phi \in[0,2 \pi)$. The SPP is TM polarized, i.e., if it propagates along the interface $z=0$ in $O x$ direction then its magnetic field, $\mathbf{H}$, is directed along $O y$ direction, $\mathbf{H}=(0, H, 0)$, and the electric field, $\mathbf{E}$, lies in the $x \mathrm{Oz}$ plane, i.e., plane of incidence, $\mathbf{E}=\left(E_{x}, 0, E_{z}\right)$. The space dependence of the SPP fields in the dielectric halfspace, $z \leq 0$, is given by the ansatz $\exp [i Q x-i p(Q) z]$, where

$$
p(\mathbf{q})=\sqrt{k^{2}-\mathbf{q}^{2}}, \quad k=\sqrt{\varepsilon_{d}} \omega / c .
$$

For dissipation-free media $p(Q)$ is pure imaginary, $p(Q)=i|p(Q)|$, so that the field amplitude decays exponentially with increasing distance from the interface $z=0$.

Recall, if the plane monochromatic electromagnetic wave with space dependence of the form

$$
\mathbf{E}, \mathbf{H} \propto \exp [i(\mathbf{q} \cdot \mathbf{r})+i p(\mathbf{q}) z], \quad \mathbf{q}=\left(q_{x}, q_{y}\right)
$$

(where and everywhere else the time dependence is supposed to be of the form $\exp (-i \omega t)$ and is omitted) is incident on the interface from the dielectric medium located at negative $z$ values, $-\infty<z<\zeta(x)$, where the surface relief, $z=\zeta(x)$, presents periodic function with period $d$, $\zeta(x+d)=\zeta(x)$, then the electromagnetic field within the dielectric medium is the sum of spatial harmonics of the form

$$
\begin{aligned}
& \mathbf{E}_{n}, \mathbf{H}_{n} \propto \exp \left[i\left(\mathbf{q}_{n} \cdot \mathbf{r}\right)-i p\left(\mathbf{q}_{n}\right) z\right], \\
& \mathbf{q}_{n}=\mathbf{q}+n \mathbf{g}, \quad \mathbf{g}=\mathbf{e}_{x} 2 \pi / d, \quad n=0, \pm 1, \pm 2, \ldots,
\end{aligned}
$$

$\mathbf{e}_{x}$ is the unit vector directed along the $O x$ axis. In other words, the diffracted field is given by the Floquet-Fourier expansion [7,9]. In (3) the sign minus before $p\left(\mathbf{q}_{n}\right)$ stays to satisfy the radiation conditions at $z=-\infty$. Restriction of the outgoing (and evanescent) waves within the whole halfspace $z \leq \zeta(x)$ corresponds to the use of the Rayleigh hypothesis [2] and is not restrictive even for rather deep gratings, see the recent discussion in [10].

Consequently, if for some specific integer $n$ the condition $\left|\mathbf{q}_{n}\right|=Q$ holds true, then for the appropriate polarization of this diffracted wave the resonance excitation of SPP takes place. It is significant that SPP is an evanescent wave and thus the magnitude of the corresponding diffracted order can exceed essentially that of the incident wave. Specifically, in the simplest geometry, when $\mathbf{q}$ is orthogonal to the grating grooves, $\mathbf{q}=(q, 0,0), q>0$, only TM component of the incident wave can excite the SPP.* Also, it deserves attention that for the modulated interface the SPP resonance centre experiences shift as compared with the "naked" condition, $\left|\mathbf{q}_{n}\right|=Q$. However, the SPP resonance in majority of experimental situations in visible and near infrared spectral regions seems to be rather evident to attribute.

We would like to underline that the Rayleigh and the resonance anomalies are related to a specific and rather sharp dependence of the field amplitudes on the wavelength and angle of incidence. They can be considered on the basis of simple qualitative treatment. The treatment of the third mentioned Wood anomaly cannot be accomplished without thorough theoretical approach. The method for considering this and other diffraction anomalies analytically was presented in [9], see also a more detailed consideration in [11-13].

\section{Grazing incidence anomaly}

In this section we present the brief summary of the results for the case of the simplest geometry which are essential for further consideration. For the TM polarization of interest the magnetic field is orthogonal to the plane of incidence and thus possesses the $y$ component only, so that for the incident wave, $\mathbf{H}^{i}$, and for the Fourier-Floquet expansion of the diffracted field, $\mathbf{H}^{D}$, we have

$$
\begin{gathered}
\mathbf{H}^{i}=\mathbf{e}_{y} H \exp [\operatorname{iqx}+i p(q) z], \\
\mathbf{H}^{D}=\mathbf{e}_{y} \sum_{n=-\infty}^{\infty} H_{n} \exp \left[i q_{n} x-i p\left(q_{n}\right) z\right], z \leq \zeta(x),
\end{gathered}
$$

where $q_{n}=q+n g$. Note, the diffracted field in (4) and below in (5) includes only outgoing (and evanescent) waves, i.e., here we use the Raylegh hypothesis [2], restricting the expansion to the terms with $z$-dependence of the form $\exp \left[-i p\left(q_{n}\right) z\right]$ only, and omitting those with $z$-dependence of the alternative form, $\exp \left[i p\left(q_{n}\right) z\right]$. This guarantees fulfillment of the boundary (radiation) conditions at $z=-\infty$. The Raylegh hypothesis is appropriate for shallow enough gratings (however, the recent investigations [7,9] have demonstrated that it works well even for rather deep gratings).

The electric field possesses the $x$ and $z$ components only, $\mathbf{E}=\left(E_{X}, 0, E_{z}\right)$, and can be easily obtained from (3) and corresponding Maxwell equation. Specifically, the diffracted field is

$$
\mathbf{E}^{D}=\sum_{n=-\infty}^{\infty} \mathbf{E}_{n} \exp \left[i q_{n} x-i p\left(q_{n}\right) z\right], z \leq \zeta(x) .
$$

* Noteworthy, in the simplest geometry the diffraction of TE and TM components of the incident wave become independent processes and thus can be considered separately. 
At the interface the total fields, $\mathbf{H}=\mathbf{H}^{i}+\mathbf{H}^{D}$, $\mathbf{E}=\mathbf{E}^{i}+\mathbf{E}^{D}$ are to obey the impedance boundary conditions [14],

$$
\mathbf{E}_{t}=\xi[\mathbf{n} \times \mathbf{H}] \text { for } z=\zeta(x),
$$

where the subindex $t$ denotes tangential to the interface component of the corresponding vector, and $\mathbf{n}$ stays for the unit vector normal to the interface and directed into the dielectric, i.e., $\mathbf{n}=-\left[\mathbf{e}_{z}-\mathbf{e}_{x} \partial \zeta / \partial x\right] / \sqrt{1+(\partial \zeta / \partial x)^{2}} \cdot{ }^{*}$ For nonmagnetic media the surface impedance $\xi$ in Gauss units is dimensionless and $\xi=\sqrt{\varepsilon_{d} / \varepsilon}$.

The relief Fourier series expansion is

$$
\begin{gathered}
\zeta(x)=\sum_{n=-\infty}^{\infty} \zeta_{n} \exp (\text { ing } x), \\
g=2 \pi / d>0, \quad \zeta_{-n}=\zeta_{n}^{*}, \quad \zeta_{0}=0 .
\end{gathered}
$$

The condition $\zeta_{0}=0$ corresponds to the specific choice of $\mathrm{Oz}$ axis origin. The Fourier series coefficients of the interface normal, $\mathbf{n}=\mathbf{n}(x)$, can be expressed in terms of $\zeta_{n}$.

Substituting into Eq. (6) the fields representations given in Eqs. (4), (5), expressing the electric field Fourier amplitudes, $\mathbf{E}_{n}$, in terms of the magnetic ones, $H_{n}$, and equating terms with equal space dependence, we arrive at the infinite system of linear algebraic equations for the transformation coefficients (TCs), $h_{n}=H_{n} / H$,

$$
\sum_{m=-\infty}^{\infty} D_{n m} h_{m}=V_{n}, \quad n=0, \pm 1, \pm 2, \ldots,
$$

where the matrix of the system, $\hat{D}=\left\|D_{n m}\right\|$, and the righthand side column vector, $\hat{V}=\operatorname{col}\left\{V_{n}\right\}$, represent functionals depending on the problem parameters, specifically, the relief $\zeta(x)$. The coefficients of the system allow infinite series expansions with respect to $\zeta_{n}$. It is of essence that strong diffraction anomalies take place for rather shallow gratings such that $k|\zeta|,|d \zeta / d x| \ll 1$, see [11-13] and below, so the expansions are very useful. For shallow gratings we can restrict series expansions of the coefficients to the main (linear) terms only, so that

$$
\begin{gathered}
D_{n m}=\left(\beta_{n}+\xi\right) \delta_{n m}-i\left(1-\alpha_{n} \alpha_{m}\right) \mu_{n-m}, \\
n, m=0, \pm 1, \pm 2, \ldots, \\
V_{n}=\left(\beta_{n}-\xi\right) \delta_{n 0}+i\left(1-\alpha_{n} \alpha_{0}\right) \mu_{n}, \quad n=0, \pm 1, \pm 2, \ldots
\end{gathered}
$$

Here $\delta_{n m}$ stays for the Kronecker delta-symbol, and

$$
\begin{gathered}
\mu_{n}=k \zeta_{n}, \quad \alpha_{n}=\alpha+n \kappa, \quad \kappa=g / k, \\
\beta_{n}=\sqrt{1-\alpha_{n}^{2}}, \quad \operatorname{Re}, \operatorname{Im} \beta_{n} \geq 0, \quad n=0, \pm 1, \pm 2, \ldots,
\end{gathered}
$$

$\alpha=\sin \theta, \theta$ denotes the incidence angle. Noteworthy, the nondiagonal elements of the matrix $\hat{D}=\left\|D_{n m}\right\|$ possess the following simple symmetry

$$
D_{n m}^{*}=-D_{m n} \text { for } n \neq m \text {. }
$$

Below we are dealing with the grazing anomalies. They correspond to specific dependences (maxima, minima) of the energetic characteristics (say, intensities) of the diffracted waves in the vicinity of the point where the incident wave or one of the diffracted waves is propagating at a small angle with respect to the interface (grazing propagation). The simplest (but of high interest) case here presents the grazing incidence, $0<\beta \ll 1(0<1-\alpha \ll 1)$. That is the specular reflected wave with necessity is the grazing one. The simplest geometry of such problem is presented in Fig. 1.

It should be emphasized, that among diffracted waves only the specular reflected one is close to the corresponding Rayleigh point, $\beta \ll 1$, and all the other waves are far enough from their branch points. That is, the only one diagonal element of the matrix $\hat{D}=\left\|D_{n m}\right\|$, namely, $D_{00}=\beta+\xi$, is small as compared with unity. Consequently, it is convenient to decompose the governing system, Eq. (8), as

$$
\begin{gathered}
\hat{\bar{D}} \hat{\bar{h}}=\hat{\bar{V}}, \\
D_{00} h_{0}+\sum_{M \neq 0} D_{0 M} h_{M}=V_{0},
\end{gathered}
$$

where and below capital indexes denote all integers except zero,

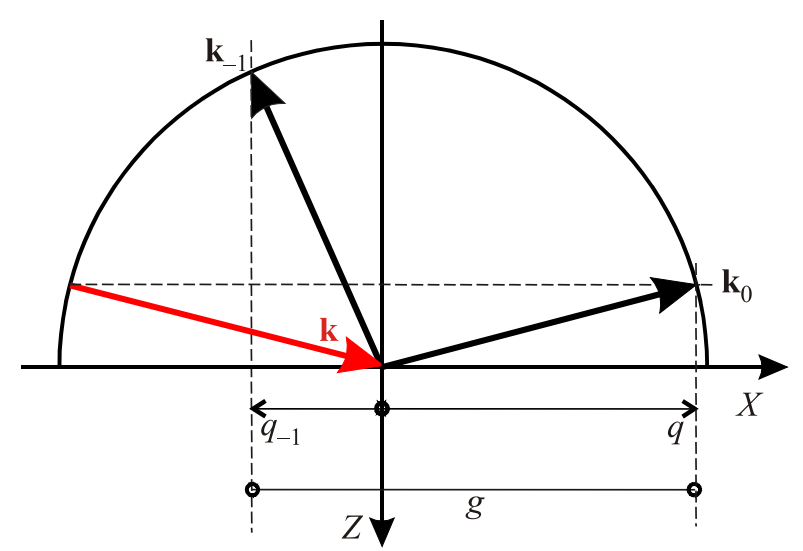

Fig. 1. Grazing incidence diffraction. The grating spacing, $d$, is supposed to be such that except the specular wave only the minus first diffraction order presents propagating wave, other diffraction orders correspond to evanescent ones, i.e., at $q \simeq k, q_{1}=q+g>k$, $q_{-1}=q-g>-k$ and $\left|q_{n}\right|>k$ for all $n \neq-1,0$.

* The following considerations can be applied to the case of plane surface of metamaterials with periodically modulated electromagnetic properties so that the surface impedance is space-periodic, $\xi=\xi(x), \xi(x+d)=\xi(x)$, cf. [11-13]. 


$$
\hat{\bar{D}}=\left\|D_{N M}\right\|, \quad N, M= \pm 1, \pm 2, \ldots,
$$

$\hat{\bar{h}}$ and $\hat{\bar{V}}$ stay for column vectors

$$
\begin{gathered}
\hat{\bar{h}}=\operatorname{col}\left\{h_{M}\right\}, \quad \hat{\bar{V}}=\operatorname{col}\left\{\bar{V}_{M}\right\}, \quad M= \pm 1, \pm 2, \ldots, \\
\bar{V}_{M}=V_{M}-D_{M 0} h_{0}, \quad M= \pm 1, \pm 2, \ldots
\end{gathered}
$$

Let us present Eq. (13) in a more explicit form as well

$$
(\beta+\xi) h_{0}-i \sum_{M \neq 0}\left(1-\alpha_{0} \alpha_{M}\right) \mu_{-M} h_{M}=\beta-\xi .
$$

The submatrix $\hat{\bar{D}}$ is diagonal dominated due to the fact that all nondiagonal elements are small as compared with unity and all diagonal ones are of order unity or greater. Thus, it can be easily inversed by means of the regular series expansion, see below. Formally, we can express all nonspecular amplitudes, $h_{M}$, in terms of the given parameters of the system and unknown at this stage amplitude $h_{0}$ as follows:

$$
\hat{\bar{h}}=\hat{\bar{D}}^{-1} \hat{\bar{V}} \text {, }
$$

or, more explicitly,

$$
h_{M}=\sum_{L \neq 0}\left[\hat{\bar{D}}^{-1}\right]_{M L} \bar{V}_{L}, \quad M= \pm 1, \pm 2, \ldots
$$

Taking into account that in accordance with Eq. (17) and Eq. (10),

$$
\begin{aligned}
\bar{V}_{L}= & V_{L}-D_{L 0} h_{0}=i\left(1-\alpha_{L} \alpha_{0}\right) \mu_{L}+i\left(1-\alpha_{L} \alpha_{0}\right) \mu_{L} h_{0}= \\
& =i\left(1+h_{0}\right)\left(1-\alpha_{L} \alpha_{0}\right) \mu_{L}, \quad L= \pm 1, \pm 2, \ldots, \quad \text { (20) }
\end{aligned}
$$

rearrange Eq. (19) as

$$
\begin{gathered}
h_{M}=i\left(1+h_{0}\right) \sum_{L \neq 0}\left[\hat{\bar{D}}^{-1}\right]_{M L}\left(1-\alpha_{L} \alpha_{0}\right) \mu_{L}, \\
M= \pm 1, \pm 2, \ldots
\end{gathered}
$$

Substituting this expression into Eq. (17) we arrive at the closed linear equation for the specular TC,

$$
\begin{gathered}
(\beta+\xi) h_{0}+\left(1+h_{0}\right) \times \\
\times \sum_{M, L \neq 0}\left[\hat{\bar{D}}^{-1}\right]_{M L}\left(1-\alpha_{0} \alpha_{M}\right)\left(1-\alpha_{L} \alpha_{0}\right) \mu_{L} \mu_{-M}=\beta-\xi .
\end{gathered}
$$

Let, for brevity,

$$
\begin{gathered}
\Gamma=\sum_{M, L \neq 0}\left[\hat{\bar{D}}^{-1}\right]_{M L}\left(1-\alpha_{0} \alpha_{M}\right)\left(1-\alpha_{L} \alpha_{0}\right) \mu_{L} \mu_{-M}, \\
\xi_{\text {eff }}=\xi+\Gamma .
\end{gathered}
$$

Then solution of Eq. (22) for $h_{0}$ can be presented as

$$
h_{0}=\frac{\beta-\xi_{\text {eff }}}{\beta+\xi_{\text {eff }}} .
$$

It is of interest that the specular TC form, Eq. (25), coincides with the corresponding Fresnel coefficient related to the unmodulated (plane) interface,

$$
R=\frac{\beta-\xi}{\beta+\xi} \text {. }
$$

For the nonspecular TCs it follows identically

$$
\begin{gathered}
h_{M}=\frac{2 i \beta}{\beta+\xi_{\text {eff }}} \sum_{L \neq 0}\left[\hat{\bar{D}}^{-1}\right]_{M L}\left(1-\alpha_{L} \alpha_{0}\right) \mu_{L}, \\
M= \pm 1, \pm 2, \ldots
\end{gathered}
$$

It is convenient to introduce subsidiary functions $U_{M}$ so that

$$
\begin{gathered}
U_{M}=\sum_{L \neq 0}\left[\hat{\bar{D}}^{-1}\right]_{M L}\left(1-\alpha_{L} \alpha_{0}\right) \mu_{L}, \quad M= \pm 1, \pm 2, \ldots \\
M= \pm 1, \pm 2, \ldots
\end{gathered}
$$

Then

$$
h_{M}=\frac{2 i \beta}{\beta+\xi_{\text {eff }}} U_{M}, \quad M= \pm 1, \pm 2, \ldots
$$

It is of essence that the coefficients $U_{M}$ experience only slow dependence on the parameters of interest in the vicinity of the point $\beta=0$, as well as the functions $\Gamma$ and $\xi_{\text {eff }}$. Noteworthy, the quantity $\Gamma$ can be expressed in terms of $U_{M}$ as

$$
\Gamma=\sum_{M}\left(1-\alpha_{0} \alpha_{M}\right) U_{M} \mu_{-M} .
$$

In what follows we are dealing with smooth and shallow gratings so here we present the main terms of the necessary functions expansions. Let

$$
\hat{\bar{D}}=\hat{\bar{B}}(\hat{\bar{I}}-\hat{\bar{T}}),
$$

where

$$
\begin{gathered}
\hat{\bar{I}}=\left\|\delta_{N M}\right\|, \quad \hat{\bar{T}}=\left\|\bar{T}_{N M}\right\|, \quad \hat{\bar{B}}=\left\|\bar{B}_{N M}\right\|, \\
N, M= \pm 1, \pm 2, \ldots, \\
\bar{T}_{N M}=\frac{i}{b_{N}}\left(1-\alpha_{N} \alpha_{M}\right) \mu_{N-M}, \quad \bar{B}_{N M}=b_{N} \delta_{N M}, \\
b_{N}=\beta_{N}+\xi, \quad N, M= \pm 1, \pm 2, \ldots .
\end{gathered}
$$

Then

$$
\hat{\bar{D}}^{-1}=(\hat{\bar{I}}-\hat{\bar{T}})^{-1} \hat{\bar{B}}^{-1}=\left[\sum_{s=0}^{\infty} \hat{\bar{T}}^{s}\right] \hat{\bar{B}}^{-1}
$$

This series expansion converges under the condition $|\hat{\bar{T}}|<1$, where || stays for the matrix norm. It is of essence that this condition is not restrictive: it allows consideration of strong anomalies, see [9,11-13] and below. Moreover, 
strong anomalies hold for $|\hat{\bar{T}}| \ll 1$. So, we can make use of the first terms of the expansion. With accuracy up to the second-order terms with respect to $\mu$,

$\left[\hat{\bar{D}}^{-1}\right]_{M L}=\left[\delta_{M L}+T_{M L}+\sum_{K \neq 0} T_{M K} T_{K L}\right] b_{L}^{-1}+O\left(\mu^{3}\right)$,

or, more explicitly,

$$
\begin{gathered}
{\left[\hat{\bar{D}}^{-1}\right]_{M L} \simeq\left[\delta_{M L}+\frac{i}{b_{M}}\left(1-\alpha_{M} \alpha_{L}\right) \mu_{M-L}-\right.} \\
\left.-\frac{1}{b_{M}} \sum_{K \neq 0} \frac{1}{b_{K}}\left(1-\alpha_{M} \alpha_{K}\right)\left(1-\alpha_{K} \alpha_{L}\right) \mu_{M-K} \mu_{K-L}\right] b_{L}^{-1} .
\end{gathered}
$$

After simple rearrangement we obtain alternative expression,

$$
\begin{aligned}
& {\left[\hat{\bar{D}}^{-1}\right]_{M L} \simeq b_{M}^{-1}\left[\delta_{M L}+\frac{i}{b_{L}}\left(1-\alpha_{M} \alpha_{L}\right) \mu_{M-L}-\right.} \\
& \left.-\sum_{K \neq 0} \frac{1}{b_{L} b_{K}}\left(1-\alpha_{M} \alpha_{K}\right)\left(1-\alpha_{K} \alpha_{L}\right) \mu_{M-K} \mu_{K-L}\right] .
\end{aligned}
$$

Consequently, up to the second-order terms it follows from Eq. (28):

$$
\begin{gathered}
U_{M} \simeq b_{M}^{-1}\left[\left(1-\alpha_{M} \alpha_{0}\right) \mu_{M}+\right. \\
\left.+i \sum_{L \neq 0} b_{L}^{-1}\left(1-\alpha_{L} \alpha_{0}\right)\left(1-\alpha_{L} \alpha_{M}\right) \mu_{L} \mu_{M-L}\right] \\
M= \pm 1, \pm 2, \ldots
\end{gathered}
$$

Noteworthy, here the second-order terms are of essence if the corresponding Fourier amplitude of the grating, $\mu_{M}$, vanishes or is anomalously small. Under this condition the anomalous effects in $M$ th diffraction order are small and thus of low interest. Therefore, below we can restrict ourselves with the linear term of $U_{M}$ expansion.

The main term of the quantity $\Gamma$ expansion is the square one,

$$
\Gamma=\sum_{M \neq 0} b_{M}^{-1}\left(1-\alpha_{0} \alpha_{M}\right)^{2}\left|\mu_{M}\right|^{2}
$$

\section{Energy flux extremes}

The solution obtained allows one to analyze in detail its dependence on the angle of incidence and all other parameters of the problem. Expressions (25), (29) describe the fast dependence of the TCs on the angle of incidence through the quantity $\beta=\cos \theta \ll 1$. Other functions entering the solution, $U_{N}$, $\xi_{\text {eff }}$, etc., are slow ones, and thus for preliminary analytical considerations can be replaced with constants relating to their values at $\beta=0$. This fact allows to perform thorough analytical investigation of the problem. Starting with the specular reflectivity

$$
\rho(\beta)=\left|h_{0}\right|^{2}=\frac{\left(\beta-\xi_{\text {eff }}^{\prime}\right)^{2}+\xi_{\text {eff }}^{\prime \prime 2}}{\left(\beta+\xi_{\text {eff }}^{\prime}\right)^{2}+\xi_{\text {eff }}^{\prime \prime 2}},
$$

one can see that it possesses specific minimal value at some point, $\beta=\beta_{\text {extr }}$, such that

$$
\beta_{\text {extr }}=\left|\xi_{\text {eff }}\right| .
$$

With high accuracy one can approximate $\xi_{\text {eff }}$ here by it value at $\beta=0$.

At the extreme point, $\beta=\beta_{\text {extr }}$, we obtain

$$
\rho=\rho_{\text {min }}, \quad \rho_{\text {min }} \equiv \rho\left(\beta_{\text {extr }}\right)=\frac{\left|\xi_{\text {eff }}\right|-\xi_{\text {eff }}^{\prime}}{\left|\xi_{\text {eff }}\right|+\xi_{\text {eff }}^{\prime}} .
$$

Here and below the prime (double prime) denotes the real (imaginary) part of the corresponding quantity. The specular TC field at the point $\beta=\beta_{\text {extr }}$ is as follows

$$
h_{0}\left(\beta_{\text {extr }}\right)=\frac{\left|\xi_{\text {eff }}\right|-\xi_{\text {eff }}}{\left|\xi_{\text {eff }}\right|+\xi_{\text {eff }}} .
$$

Noteworthy, the analogous minimum for TM polarized wave incidence exists for unmodulated interface, $\Gamma=0$, $\xi_{\text {eff }} \Rightarrow \xi$ (when $h_{0}$ coincides with the corresponding Fresnel reflection coefficient $R$ ) and is discussed in [14]. This minimum is analogous to the reflectivity minimum from dielectric medium existing under Brewster angle incidence (when the reflected and transmitted waves are propagating at a right angle) [14]. In view of the fact that for $|\varepsilon| \gg 1$ (which is typical for good metals up to the frequencies of the visible range), the normal to the interface component of the wavevector in metal prevails essentially the tangential one, so the wave in metal can be formally considered as orthogonal to the interface. Consequently, for grazing incidence the reflected from the metal wave is approximately orthogonal to the "transmitted" one. Recall, the Brewster angle of incidence, $\theta_{\mathrm{Br}}$, is defined as

$$
\sin \theta_{\mathrm{Br}}=\sqrt{\varepsilon} / \sqrt{\varepsilon+1},
$$

so that for $|\varepsilon| \rightarrow \infty$ one obtains $\theta_{\mathrm{Br}} \simeq \pi / 2$.

The specular reflectivity minimum, Eq. (41), becomes deep for relatively high effective losses, i.e., for $\xi_{\text {eff }}^{\prime}$ comparable with $\left|\xi_{\text {eff }}^{\prime \prime}\right|$. It worth to point out here that $\xi_{\text {eff }}^{\prime}$ includes both the dissipative and radiative losses relating to the quantities $\xi^{\prime}$ and $\Gamma^{\prime}$, respectively. The quantity $\Gamma^{\prime}$ is mainly caused by outgoing (propagating) waves. On the contrary, $\rho_{\text {min }}$ approaches unity at vanishing lossess, $\xi_{\text {eff }}^{\prime} \rightarrow 0$. Therefore, the effect of the specular reflection suppression under consideration is mainly attributed to the cumulative (both active and radiative) losses maximum, $\mathrm{cf}$. 
[15]. However, as it is shown below the point $\beta=\beta_{\text {extr }}$ corresponds not only to the specular reflection minimum, but results in well expressed maximal nonspecular efficiencies. Evidently, if the only propagating diffracted wave is the specular one, then the grazing minimum is with necessity accompanied by maximal absorption. Noteworthy, the reflectance minimum under grazing incidence can correspond to essential redirection of the energy into nonspecular diffraction channels corresponding to propagating waves even for shallow gratings. Below this thesis is illustrated for the simplest case when in addition to the specular wave only one diffracted order corresponds to propagating (outgoing) wave. It can be realized if $1+\alpha>\kappa>1$, the minus first order presents propagating wave, $\beta_{-1}>0$, and $\beta_{n}$ with $n \neq-1,0$ are pure imaginary. In what follows we consider that $\beta_{-1}$ is of order unity, so that the minus first diffraction order is far from its Rayleigh point.*

It is of interest that normalized intensities of the propagating diffraction orders,

$$
\rho_{N}=\left|h_{N}\right|^{2} \frac{\operatorname{Re}\left(\beta_{N}\right)}{\beta}=4 W\left|U_{N}\right|^{2} \operatorname{Re}\left(\beta_{N}\right),
$$

present strongly nonmonotonic $\beta$ functions in accordance with the fast dependence of the subsidiary function introduced, $W=W(\beta)$,

$$
W(\beta)=\frac{\beta}{\left(\beta+\xi_{\text {eff }}^{\prime}\right)^{2}+\left(\xi_{\text {eff }}^{\prime \prime}\right)^{2}} .
$$

It is easy to see that $W(\beta)$ achieves its maximal value, $W_{\text {max }}$, strictly at the point $\beta=\beta_{\text {extr }}$, and

$$
W_{\max }=W\left(\beta_{\text {extr }}\right)=\frac{1}{2\left(\left|\xi_{\text {eff }}\right|+\xi_{\text {eff }}^{\prime}\right)} \gg 1 .
$$

That is, all intensities simultaneously achieve their maximal values at the point $\beta=\beta_{\text {extr }}$,

$$
\begin{gathered}
\rho_{N, \max }=\rho_{N}\left(\beta_{\text {extr }}\right)=\frac{2\left|U_{N}\right|^{2}}{\left|\xi_{\text {eff }}\right|+\xi_{\text {eff }}^{\prime}} \operatorname{Re}\left(\beta_{N}\right), \\
N= \pm 1, \pm 2, \ldots
\end{gathered}
$$

It seems necessary to check that, first, the total energy flux outgoing with the propagating waves does not prevail that of the incident wave, i.e.,

$$
\sum_{N} \rho_{N} \leq 1
$$

where $\rho_{0}$ stays for $\rho$. The difference between the sum and unity is nothing else than the active losses (per unit area). This inequality is to be true under rather general conditions, specifically for such $\beta$ and $\kappa$ values that we are far from anomalies relating to all diffraction orders escept the specular one. If the active losses are absent, then the inequality transforms into the equality. In the specific case of short-period gratings, such that $\kappa>2$ all diffracted orders except the zeroth one with necessity correspond to evanescent waves. Therefore, the strong specular reflectivity suppression is accompanied by maximal absorption. Underline, this conclusion is true under rather specific consitions and does not describe general case contrary to the statement of [15].

For the specific case shown in Fig. 1 only two diffraction orders correspond to propagating waves; the specular and the minus first ones. Consider this specific subcase in more detail. Suppose additionally that the grating is harmonic, i.e.,

$$
\mu_{1}=\mu_{-1}=a>0, \quad \mu_{n}=0 \text { for }|n| \geq 2 .
$$

Then

$$
\begin{aligned}
& \Gamma \simeq a^{2}\left[\frac{\left(1-\alpha_{0} \alpha_{1}\right)^{2}}{b_{1}}+\frac{\left(1-\alpha_{0} \alpha_{-1}\right)^{2}}{b_{-1}}\right] \simeq \\
& \simeq a^{2}\left[-i \frac{\left(1-\alpha_{0} \alpha_{1}\right)^{2}}{\left|\beta_{1}\right|}+\frac{\left(1-\alpha_{0} \alpha_{-1}\right)^{2}}{\beta_{-1}}\right],
\end{aligned}
$$

where it is taken into account that $\left|\beta_{ \pm 1}\right| \gg|\xi|$. In the geometry under discussion

$$
1-\alpha_{0} \alpha_{ \pm 1}=1-\alpha(\alpha \pm \kappa)=\beta^{2} \mp \alpha \kappa \simeq \mp \kappa .
$$

Consequently, $\Gamma$ can be simplified as

$$
\Gamma \simeq \kappa^{2} a^{2}\left[\frac{1}{\beta_{-1}}-i \frac{1}{\left|\beta_{1}\right|}\right] .
$$

As far as

$$
\beta_{ \pm 1}^{2}=1-(\alpha \pm \kappa)^{2}=\beta^{2} \mp 2 \alpha \kappa-\kappa^{2} \simeq-\kappa^{2} \mp 2 \kappa,
$$

we can express $\Gamma$ in terms of the dimensionless parameter of the problem, $\kappa$, only, neglecting slow dependence on the angle of incidence,

$$
\Gamma \simeq \kappa^{2} a^{2}\left[\frac{1}{\sqrt{\kappa(2-\kappa)}}-i \frac{1}{\sqrt{\kappa(2+\kappa)}}\right] .
$$

In view of the fact that the specular reflectivity possesses rather expressed minimal value then for low active losses the incoming energy is to be redirected in other propagating waves. The most interesting case allowing to obtain rather strong grazing anomalies presents such one that

$$
|\Gamma| \gg|\xi|
$$

* Alternative case is of interest also, resulting in strong GA as well. The specific case when GA is accompanied by SPP anomaly relating to some other diffraction order is also of interest. These cases correspond to the double and combined anomalies and will be considered in forthcoming papers. 
i.e., the case when the effective impedance is mostly caused by the diffraction rather than the medium properties. ${ }^{*}$ It is of essence that the supposition presented in Eq. (53) does not contradict the shallow character of the grating, $|\Gamma| \sim a^{2} \ll 1$, in view of the surface impedance smallness, $|\xi| \ll 1$. The characteristic value of the dimensionless grating height, $a_{\mathrm{cr}}$, is small, $a_{\mathrm{cr}}=\sqrt{|\xi|} \ll 1$. Under Eq. (53) condition $\rho_{-1, \max }$ and $\rho_{\min }$ can be rewritten as

$$
\rho_{-1, \max } \simeq \frac{2 \Gamma^{\prime}}{|\Gamma|+\Gamma^{\prime}}, \quad \rho_{\min } \simeq \frac{|\Gamma|-\Gamma^{\prime}}{|\Gamma|+\Gamma^{\prime}},
$$

or, in view of Eq. (50),

$$
\rho_{-1, \text { max }} \simeq \frac{2\left|\beta_{1}\right|}{\sqrt{\left|\beta_{1}\right|^{2}+\beta_{-1}^{2}}+\left|\beta_{1}\right|}, \quad \rho_{\text {min }} \simeq \frac{\sqrt{\left|\beta_{1}\right|^{2}+\beta_{-1}^{2}}-\left|\beta_{1}\right|}{\sqrt{\left|\beta_{1}\right|^{2}+\beta_{-1}^{2}}+\left|\beta_{1}\right|} .
$$

Bearing in mind Eq. (51) we proceed

$$
\rho_{-1, \max } \simeq \frac{2 \sqrt{2+\kappa}}{2+\sqrt{2+\kappa}}, \quad \rho_{\min } \simeq \frac{2-\sqrt{2+\kappa}}{2+\sqrt{2+\kappa}} .
$$

That is, for rather deep gratings, $a \gg a_{\text {cr }}$ (but $a \ll 1$ ) the energy redistribution does not depend on their height, $\rho_{-1, \max }$ and $\rho_{\min }$ depend on the geometrical parameters and the wavelength through the dimensionless combination $\kappa=\lambda / d$ only.

It is easy to see that within the accuracy indicated $\rho_{-1, \max }+\rho_{\min }=1$. That is, all incident energy is redistributed only between two propagating waves, while the active losses are negligibly low.

As far as we know the grazing diffraction anomaly under discussion was not considered earlier. However, in $[6,16]$ one can find the related anomalous effect arising for such parameters of the diffraction problem that some diffracted order corresponds to the grazing wave propagating at the specific grazing angle. The anomaly consists in highly enhanced efficiency of this wave accompanied by deep suppression of the specular reflection. It is worth noting that this grazing wave enhancement is related to the problem under consideration by the reciprocity theorem $[17,18]$. Namely, reversing the propagation direction of the minus first order diffracted wave in Fig. 1 we arrive at the reciprocal diffraction problem. In the latter the corresponding minus first order is related to the grazing wave propagating in the opposite direction to the incident wave in the primordial problem. In more detail the reciprocity approach will be discussed in forthcoming papers.

We also present an illustration of other anomalous effects relating to the interface of metal and isotropic dielectric (vacuum, for simplicity). It is convenient to consider

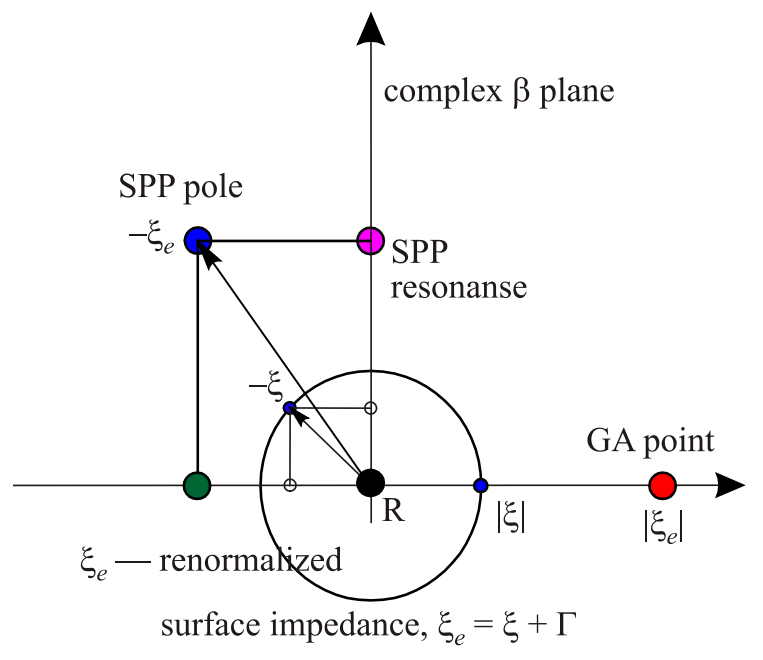

Fig. 2. Beta-plane for some diffraction order. Only vicinity of the corresponding Rayleigh point (that is of main interest in view of the diffraction anomalies) is shown. With the change of the parameters of the problem, the $\beta$ value can be either pure real positive (propagating wave), or pure imaginary (evanescent wave). These cases are separated by the Rayleigh (branch) point, $R$, $\beta=0$. Other characteristic points, $\beta_{S P P}=-i \operatorname{Im}\left(\xi_{e}\right)$ and $\beta_{G A}=\left|\xi_{e}\right|$ relating to SPP resonance and to the grazing anomaly (GA) are shown by circles. Here $\xi_{e}$ is shortened form of $\xi_{\text {eff }}$. If $\beta$ corresponds to the incident wave, then it is pure real and only GA point is actually of interest.

the effects in terms of the dimensionless normal component $\beta$ of the corresponding diffraction order. This quantity can be pure real or pure imaginary belonging to positive half-axis for both cases. The point $\beta=-\xi_{\text {eff }}$ in the $\beta$ plane, Fig. 2, corresponds to the relating diffraction order pole caused by the surface plasmon polariton mode.

\section{Conclusion}

It is shown that the diffraction of TM polarized wave at a high reflecting gratings under grazing incidence can result in deep suppression of the specular reflection accompanied by considerable redirection of the incoming energy to other propagating diffracted waves. This phenomenon becomes more pronounced at low temperatures due to small dissipation. The detailed theoretical analysis of the problem is presented on the basis of appropriate analytical approach. The diffraction anomaly considered in the paper is of general character and can take place for other wave types under appropriate conditions (high contrast of the adjacent media properties). In particular, it can hold at the interface of ordinary dielectric media and for left-handed media as well. Analogous anomaly does exist and is well expressed for magnetic high-contrast media interface for TE polarization.

* Specifically, such condition holds within approximation of ideal metal that is valid in the long wavelength region (low frequency). 


\section{Acknowledgment}

The author wishes to acknowledge the constructive comments by I.S. Spevak.

1. R.W. Wood, Philos. Mag. 4, 396 (1902).

2. Lord Rayleigh, Philos. Mag. 14, 60 (1907); Proc. Roy. Soc. A 79, 399 (1907).

3. U. Fano, J. Opt. Soc. Amer. 31, 213 (1941).

4. A. Hessel and A.A. Oliner, Appl. Opt. 4, 1275 (1965).

5. R.W. Wood, Phys. Rev. 48, 928 (1935).

6. G.M. Gandelman and P.S. Kondratenko, JETP Lett. 38, 246 (1983).

7. R. Petit and M. Neviere, Light Propagation in Periodic media. Differential Theory and Design, Marcel Dekker Publisher, New York (2003).

8. O. Takayama, L.-C. Crasovan, S.K. Johansen, D. Mihalache, D. Artigas, and L. Torner, Electromagnetics 28, 126 (2008).

9. A.V. Kats and V.V. Maslov, JETP 62, 496 (1972).

10. A.V. Tishchenko, Opt. Express 17, 17102 (2009).

11. A.V. Kats, P.D. Pavitskii, and I.S. Spevak, Radiophys. and Quantum Electronics 35, 163 (1992).

12. A.V. Kats, P.D. Pavitskii, and I.S. Spevak, JETP 78, 79 (1994).

13. A.V. Kats and I.S. Spevak, Phys. Rev. B 65, 195406 (2002).

14. L.D. Landau and E.M. Lifshits, Electrodynamics of Continuous Media, Pergamon, Oxford (1977).

15. E.K. Popov, L.B. Mashev, and E.G. Loewen, Appl. Opt. 28, 970 (1989).

16. M. Tymchenko, V.K. Gavrikov, I.S. Spevak, A.A. Kuzmenko, and A.V. Kats, Appl. Phys. Lett. 106, 261602 (2015).

17. R.J. Potton, Rep. Prog. Phys. 67, 717 (2004).

18. A.A. Kuzmenko and A.V. Kats, Intern, Young Scientists Forum on Applied Physics (YSF-2015), 29 Sept.-2 Oct., 2015, Dnipropetrovsk, Ukraine.

\section{Електромагнітні аномалії при ковзному розповсюдженні. Екстремуми потоків енергії}

\section{О.В. Кац}

Ретельно проаналізовано дифракцію електромагнітних хвиль на поверхнях $з$ періодичними структурами, що супроводжується великими аномальними ефектами. Розглянуто межі поділу середовищ з високим контрастом властивостей. Дослідження обмежено випадком ТМ поляризації хвилі, що падає на межу (магнітне поле перпендикулярне до площини падіння), та найпростішою геометрією, коли площина падіння ортогональна до штрихів гратки. Найбільшу увагу приділено максимам та мінімумам густини потока енергії, що супроводжують ковзне розповсюдження в деякому дифракційному порядку. Обговорюється зв'язок з іншими аномаліями, як релеївськими, так і резонансними.

Ключові слова: дифракція, хвиля, аномалія, резонанс, гратка, потік.

\section{Электромагнитные аномалии при скользящем распространении. Экстремумы потоков энергии}

\section{А.В. Кац}

Детально проанализирована дифракция электромагнитных волн на поверхностях с периодическими структурами, сопровождаемая сильными аномальными эффектами. Рассматриваются границы раздела сред, отличающихся высоким контрастом свойств. Исследование ограничено случаем ТМ поляризации падающей на границу волны (магнитное поле ортогонально плоскости падения) и простейшей геометрией, когда плоскость падения ортогональна штрихам решетки. Наибольшее внимание уделено максимумам и минимумам плотности потока энергии, сопровождающими скользящее распространение в некотором дифракционном порядке. Обсуждается связь с другими аномалиями, как рэлеевскими, так и резонансными.

Ключевые слова: дифракция, волна, аномалия, резонанс, решетка, поток. 